\title{
Suppressed Dicrotic Notch Pulse Classifier Design
}

\author{
Bhaskar Thakker and Anoop Lal Vyas
}

\begin{abstract}
Radial pulse signals have been found to be popular in ancient culture due to its simple, non invasive and effective approach for diagnosis. Traditional Chinese Medicine defines three pulse points on radial artery of both the hands for the assessment of health associated with internal body organs. An abnormal pulse pattern identified in the subjects of Prostate Enlargement is discussed with its characteristics. The feature vector for this abnormal pulse pattern and healthy pulse patterns are derived from power spectral density of the pulse signal. Dimensionality of the feature vector is reduced by ranking the frequency components according to their classification power. Binary pulse classifiers for the classification of such abnormal pulse pattern from the healthy pulse patterns are designed. Linear and Quadratic binary pulse classifiers give $\mathbf{8 9 . 0} \%$ and $\mathbf{8 9 . 2 \%}$ classification accuracy for group of 5 and 4 initial ranked frequency components respectively.
\end{abstract}

Index Terms - Suppressed Dicrotic Notch, Traditional Chinese Medicine, Ranked Features, Binary Pulse Classifier.

\section{INTRODUCTION}

Highlight Radial pulse analysis is popular as a complementary diagnosis approach due to its simple and non invasive nature. This complementary approach informally known as "Radial Pulse Analysis" is being practiced in China India and some of the other parts of world. It gives distinct significance to three pulse pressure signals observed over the radial artery of the two hands. Traditional Chinese Medicine (TCM) correlates these pulse signals observed with light as well as deep pressure with several body organs like stomach, liver, spleen, gall bladder and intestines in the Gastro Intestinal Tract (GIT), kidney and bladder in the Urinary Tract (UT), heart and lungs [1,2].

Human body consists of complex arterial structure, which starts from aorta, delivering oxygenated blood to various organs. As the arteries get divided into branches, they become narrower and result into thin arterioles and capillaries prior to the termination in the organs. There are variations in the thickness of the inner layer as well as diameter of the arteries at various peripheral sites. Forward incident pulse wave established by left ventricle in heart gets reflected from each bifurcation in this arterial structure as well as from peripheral organs. Progressive appearance of the reflected wave results into establishment of an average pulse character. Fig. 1 shows such an average pulse signal

Manuscript received March 21, 2011. This work was partially supported by the Indo-UK collaborative project on "Mobile Communications for improving monitoring of Heart Disease and Diabetes" under the UKIERI program of British Council.

Bhaskar Thakker is a research scholar in Instrument Design Development Centre (IDDC) at Indian Institute of Technology, Delhi, India (91-11-26596741; e-mail: thakkar id@student.iitd.ac.in).

Anoop Lal Vyas is a Professor in Instrument Design Development Centre (IDDC) at Indian Institute of Technology, Delhi, India (91-11-26591442; e-mail: alvyas@iddc.iitd.ac.in). established due to combination of forward and reflected pulse waves. The parameters like timing and strength of reflected wave play important role in deciding the pulse character. These parameters depend upon not only on the arterial stiffness but also on the health status of the organs. These characteristics of the reflected wave can be seen in the falling segment of the average pulse wave. The falling segment of the pulse can therefore play an important role in providing the health status.

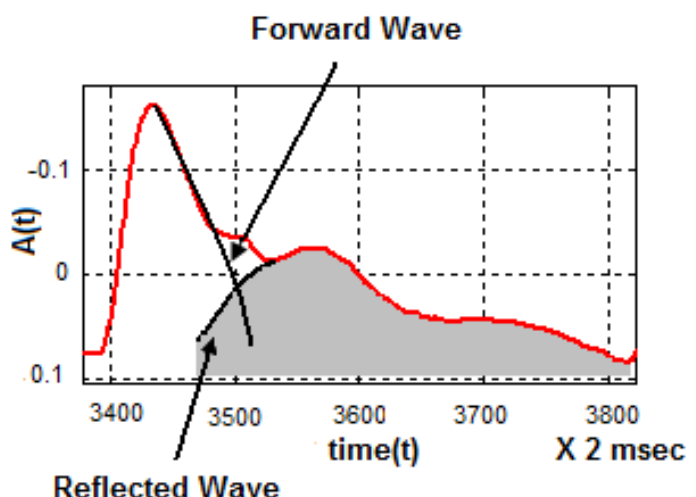

Figure 1 Pulse pressure signal phenomena

TCM believes that three radial pulse signals carry unique information about the health status. The pressure applied on the pulse point, pulse morphology at these pressures and their variations among pulse points are required to be investigated to identify the abnormal health conditions. Prime importance has been given to the applied contact pressure along with other pulse parameters like rate, rhythm, force and volume in pulse analysis. The analysis of pulse at superficial and deep contact pressure, in terms of its morphological variations can help in identifying the health status of body organs. The body organs that can be examined with three radial pulse points of both the hands have been shown in Table I.

TABLE I. ORGAN PULSES ACCORDING TO TCM

\begin{tabular}{|c|c|c|c|c|c|}
\hline \multicolumn{2}{|c|}{ Left Hand Pulse Points } & \multicolumn{3}{c|}{ Right Hand Pulse Points } \\
\hline LP1 & LP2 & LP3 & RP1 & RP2 & RP3 \\
\hline $\begin{array}{c}\text { Small } \\
\text { Intestine }\end{array}$ & $\begin{array}{c}\text { Gall } \\
\text { Bladder }\end{array}$ & Bladder & Colon & Stomach & $\begin{array}{c}\text { Triple } \\
\text { Energizer }\end{array}$ \\
\hline Heart & Liver & Kidney & Lungs & Spleen & Pericardium \\
\hline
\end{tabular}

The research articles on this subject majorly describe the commonly visible pulse patterns like moderate, slippery, taut, unsmooth and hollow pulse signals and approaches to classify them $[3,4,5]$. These pulses mainly refer in their falling segments. It is, therefore important to study the characteristics of falling pulse segment in abnormal health conditions. Power spectral density of the pulse signal in abnormal subjects mentions that the energy associated with higher frequency components elevates $[6,7,8,9]$. The studies so far do not discuss identification of specific 
abnormal pulse patterns and their association with health status.

According to TCM, the proximal radial pulse point of left hand carries information about Urinary Tract Infection (UTI) related problems. This pulse point helps in investigating the disorders associated with kidney, bladder, and prostate kinds of organ in Urinary Tract. The pulse signal for the group of patients suffering from Prostate Enlargement, are studied and an abnormal pulse pattern observed at deep pressure has been reported in our previous work [10]. In this paper a binary pulse classifier, which classifies a pulse into either a Normal or a Prostate pulse is proposed. Classifier is designed using Linear as well as Quadratic Discriminant classifier based on the optimum ranked features derived from frequency domain transformation of the pulse.

\section{PAPER PULSE SIGNAL ACQUISITION AND PROSTATE PULSE DISCRIPTION}

\section{A. Radial Pulse Acquisition and pre-processing}

A pressure sensor capable of measuring up to $350 \mathrm{mmHg}$ of pressure is used for acquiring radial pulse. The pulse signal is amplified and filtered to obtain a clean signal. The signal is sampled at $500 \mathrm{~Hz}$ with the help of USB 6009 Data Acquisition system from National Instruments. Since the radial pulse contains most of the signal in the lower frequency band, the signal is filtered with the help of a low pass filter having cut off frequency of $40 \mathrm{~Hz}$. The signal contains baseline fluctuations due to motion artifacts and respirations of the person. This gives rise to low frequency components in the range of 0 to $0.5 \mathrm{~Hz}$. To eliminate these low frequency components, the pulse signal is decomposed up to eighth level using Daubichies wavelet transform 'DB8'. The reconstructed signal from eighth level wavelet approximation coefficients (CA8) representing these fluctuations is subtracted from original pulse series to achieve a pulse signal showing only dynamic nature.

\section{B. Prostate Pulse Pattern}

The deep pressure is defined as the pressure exerted on the pulse point prior to the complete obstruction of the artery. In radial pulse signal, dicrotic notch in falling segment is visible due to closing action of aortic valve in the heart [11]. The reflected wave from peripheral organs elevates the forward wave generated by the heart and establishes an average pulse signal in the arteries. The characteristics of reflected wave decide the overall pulse morphology. The reflected wave in abnormal health conditions may be late or early as well as strong or weak. A strong reflection elevates the dicrotic notch resulting in increasing the pulse volume. In healthy subjects it has been found that the dicrotic notch has tendency to elevate upwards when the pressure on the pulse point is increased from light to deep. Further the dicrotic notch is seen well above the onset of the pulse point. This ultimately gives pulse morphologies shown in Fig. 2 for healthy subjects, formally known in the research literature with Taut, Slippery and Moderate pulse [4].

Prostate Enlargement is a kind of disorder found in male subjects, in which the size of prostate gland increases from its normal size of 20 to 30 cubic centimeters (cc). The prostate size exceeding beyond $30 \mathrm{cc}$ is referred as Prostate Enlargement. In the task of pulse collection, the patient is examined by the practitioner with the ultrasound report stating presence of prostate enlargement. For all the eight such subjects suffering from Prostate Enlargement, it has been found that at deep pressure for proximal pulse point of left hand, the dicrotic notch remains suppressed below the onset of the pulse as shown in Fig. 3. An interesting observation is that six subjects out of eight show elevation of dicrotic notch for the other two pulse points. The reflected pulse wave in this case is weak in the sense that it does not help in elevating the dicrotic notch resulting in the suppression of dicrotic notch. This observation is reported in our previous work [10].

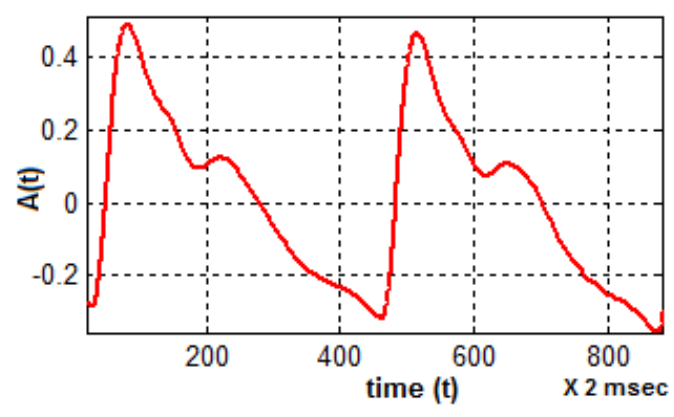

(a) Taut Pulse

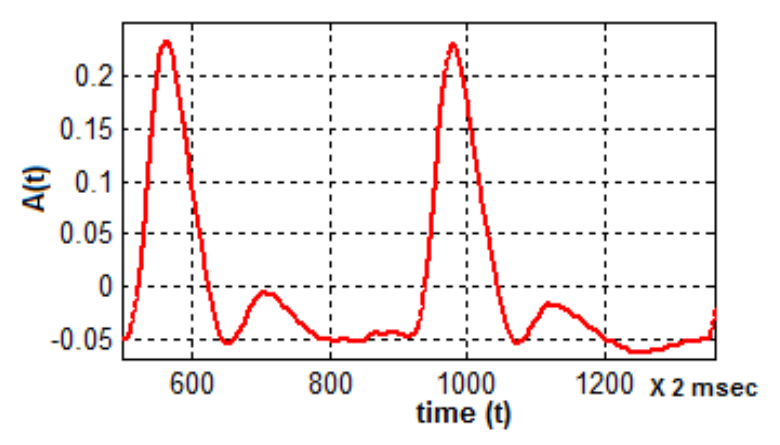

(b) Slippery Pulse

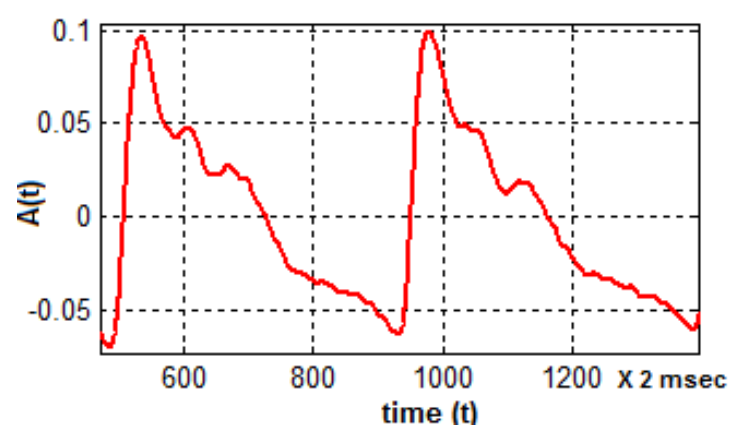

(c) Moderate Pulse

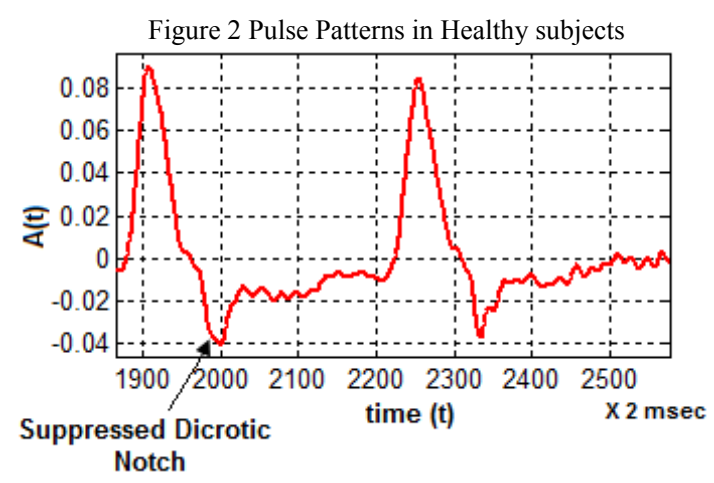

Figure 3 Suppressed Dicrotic Notch for Prostate Enlargement disorder 


\section{PULSE CLASSIFIER DESIGN}

A binary classifier classifies the pulse in one of the two categories. Feature extraction and dimensionality reduction for the features are important steps in designing a classifier. Dimensionality of the features is reduced by removing redundant features while retaining the significant features. Frequency domain features are derived here for the pulse signal at deep pressure and the features are reduced after ranking the optimum features.

\section{A. Feature Extraction}

During the pulse acquisition, the pulse strength has found to be different in the subjects due to biological status of the body. To compare the features of pulse signal having different amplitude, normalization is required to be carried out. In the task of identifying a pulse pattern, the morphology associated with the pulse signal $\mathrm{y}(\mathrm{n})$ is of prime importance to be retained. The mean subtracted pulse series is normalized to its standard deviation as shown in (1).

$$
x(n)=\frac{y(n)-\overline{y(n)}}{\sigma_{y(n)}}
$$

A healthy group of 18 subjects in the age group of 20 to 25 years has been defined. The pulse patterns for this group of subjects follow the morphologies shown in Fig. 2. An unhealthy group of 8 subjects having prostate enlargement is defined in which the pulse pattern follow the morphologies shown in Fig. 3. Normalized pulse signal of 3000 samples is transformed to frequency domain with the help of Fourier Transformation. Prior to transformation a Hamming window of 1500 sample length is used to reduce spectral leakage. Prior to windowing the signal is padded with zeros up to 2048 samples. This gives frequency resolution of $0.244 \mathrm{~Hz}$ for 500 $\mathrm{Hz}$ sampling rate. The length is chosen based on minimum resolution required as well as avoiding too many frequency components to reduce the amount of redundancy. Power spectral density with the help of $99 \%$ overlapping windows has been calculated using (2), (3) and (4).

$$
\begin{gathered}
\mathrm{x}_{\mathrm{i}}(\mathrm{n})=\mathrm{x}(\mathrm{n}+(\mathrm{i}-1) \mathrm{S}), \mathrm{i}=1,2, \ldots . . \mathrm{D} ; \mathrm{n}=0,1,2, \ldots . . \mathrm{N}-1 \\
\mathrm{X}^{\mathrm{i}}(\mathrm{k})=\sum_{\mathrm{n}=0}^{\mathrm{N}-1} \mathrm{x}(\mathrm{n}) \cdot \operatorname{window}(\mathrm{n}) \mathrm{e}^{-\frac{\mathrm{j} 2 \pi \mathrm{kn}}{\mathrm{N}}} \\
\mathrm{P}(\mathrm{k})=\frac{1}{\mathrm{D}} \sum_{\mathrm{i}=1}^{\mathrm{D}} \mathrm{X}^{\mathrm{i}}(\mathrm{k})
\end{gathered}
$$

where ' $\mathrm{i}$ ' is number of segments, ' $\mathrm{N}$ ' is the length of segment, ' $\mathrm{D}$ ' is total number of segments and window(n) is Hamming window. $\mathrm{P}(\mathrm{k})$ is the average power spectral density of all the overlapping segments. Power spectral densities of both the groups have been derived for the pulse patterns shown in Fig. 2 and 3. These frequency coefficients are used as features for the classification purpose.

\section{B. Dimensionality Reduction for pulse features}

The power spectral density of the pulse signal for single normal and abnormal subject is shown in Fig. 4. Most of the pulse energy has been found to be distributed in $0 \mathrm{~Hz}$ to $10 \mathrm{~Hz}$ frequency bands. Beyond $10 \mathrm{~Hz}$, the energy associated with the frequency components falls below $-30 \mathrm{db}$. The energy before $0.5 \mathrm{~Hz}$ is present mainly due to motion artifacts and baseline fluctuations. Hence the frequency coefficients between $0.5 \mathrm{~Hz}$ to $10 \mathrm{~Hz}$ are retained. Out of these frequency components, it is desired to identify those frequency components which can provide reasonably good classification accuracy among the two groups.

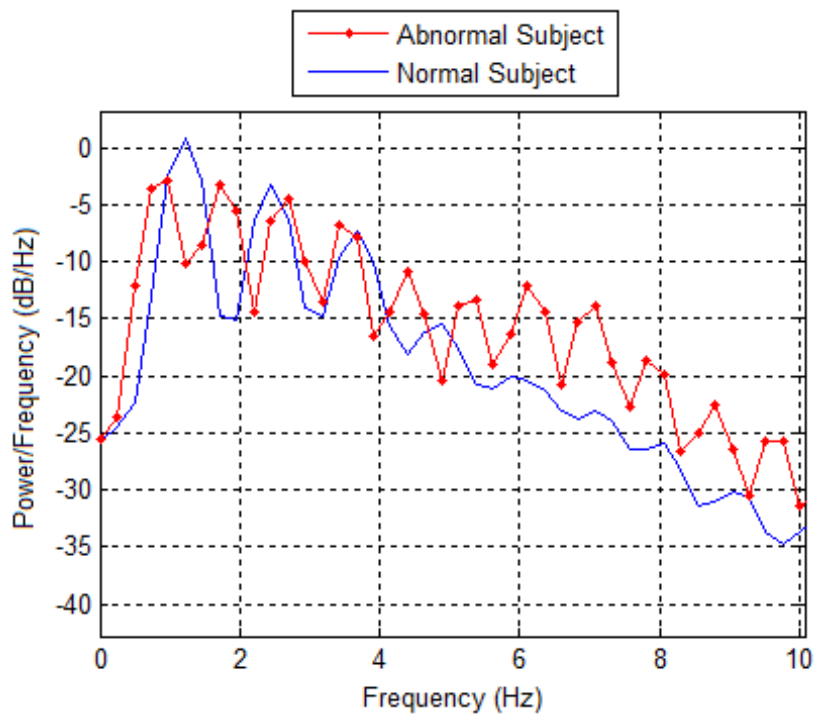

Figure 4 Power Spectral Density of single Normal and Abnormal subject

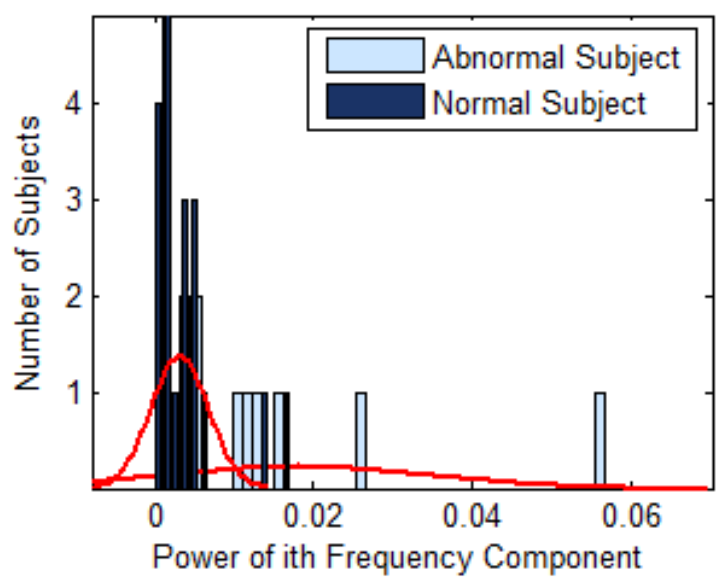

Figure 5 Ranking of ith frequency component

All the machine learning problems suffer with the curse of dimensionality. The higher amount of dimensionality associated with the feature space in the training data set results in to poor generalization. Under such situations the classifier is getting trained to identify very specific features of the patterns resulting into overfitting. Higher dimension also raises the complexity associated with the classifier in terms of training time as well as memory requirements. Hence it is always desired to identify the most important features that improve the classifier performance. Retaining only such limited features helps in achieving generalization as well as simple classifier design.

The power spectral densities in the case of both the groups show redundancy for the frequency components. Hence dimensionality reduction for the same is required. The classification power associated with each frequency 
component in the range of $0.5 \mathrm{~Hz}$ to $10 \mathrm{~Hz}$ is required to be examined. A histogram for energy contents of every frequency component corresponding to all the subjects of both the groups is derived as shown in Fig. 5. A statistical two sample t-test is carried out to compare the means of two groups. The procedure is following an iterative pair wise coupling of the frequency components. The frequency components having higher statistical power to discriminate between the two groups are ranked in the order of their classification power. Combinations of ranked features are then used as optimum feature vector for classification task.

\section{Linear and Quadratic Pulse Classifier}

A Linear Discriminant function is defined as:

$$
g(x)=w_{0}+\sum_{i=1}^{d} w_{i} x_{i}
$$

where coefficients ' $w_{i}$ ' are the components of weight vector ' $w$ ' and ' $x$ ' is a ' $d$ ' dimensional feature vector derived from the ranked frequency components of the pulse signal. Linear Discriminant classifier requires $[1+d] M$ coefficients for ' $d$ ' dimensional feature vector and ' $\mathrm{M}$ ' class problem. Linear Discriminant function gives hyperplanes in the form of classification boundary.

Another classifier using Quadratic Discriminant function utilizes additional term for the product of pairs of feature vector elements of ' $x$ ' [12]. A Quadratic Discriminant function is defined as:

$$
g(x)=w_{0}+\sum_{i=1}^{d} w_{i} x_{i}+\sum_{i=1}^{d} \sum_{j=1}^{d} w_{i j} x_{i} x_{j}
$$

Using Quadratic Discriminant classifier, with ' $d$ ' dimensional feature vector for ' $M$ ' class problem, number of weight/coefficients required will be $\left[1+d+\frac{d(d+1)}{2}\right] M$. Quadratic discrimination function gives the decision boundary in the form of hyper-quadratic structure, which can help in creating non linear decision boundary.

In both of these classifiers distinct value of $\mathrm{g}(\mathrm{x})$ is chosen for each class and weight vector ' $\mathrm{w}$ ' is trained with the help of pulse feature vector ' $x$ '. The aim of learning a classifier is to estimate the parameters ' $\mathrm{w}$ ' and $\mathrm{w}_{0}$ associated with the hyperplane. A cost function $\mathrm{J}(\mathrm{w})$ as a performance measure of the learned weight vector is defined as given in (7) for all the data points which are misclassified.

$$
J(w)=\sum_{x \in D}\left\{y(x)-w^{T} x\right\}^{2}
$$

where, D is the subset of data which is misclassified, for a given ' $w$ ' and $y(x)$ is the actual output associated with the data ' $x$ '. For non linear separable case $J(w)$ will always be finite and it is desired to find weight vector ' $w$ ' with minimum cost function. Gradient descent method as given in (8) is used to learn the weight vector to give a final solution for hyperplane.

$$
w(t+1)=w(t)-\eta_{t} \frac{\partial J(w)}{\partial(w)}
$$

where, $\eta_{t}$ is the learning rate and $\mathrm{w}(\mathrm{t}+1)$ is the new updated weight vector. Iteratively ' $\mathrm{w}$ ' is modified such that $\mathrm{J}(\mathrm{w})$ approaches towards zero.

\section{PERFORMANCE EVALUATION OF CLASSIFIER}

In supervised learning the overall database containing the features of all the pulse categories is randomly divided in training and validation data sets. The classifier is trained with training data sets and the performance of the classifier for validation data set is derived. In ' $\mathrm{m}$ ' - fold cross validation technique, the overall database is divided into ' $\mathrm{m}$ ' equal parts. Each part at a time is used as a validation data set and rest of the ' $\mathrm{m}-1$ ' parts are used for training purpose. These give the classifier results for ' $\mathrm{m}$ ' different training and validation data sets. The performance of a classifier is derived by averaging the results of ' $m$ ' validation data sets. The results derived in this manner better resembles the performance of classifier for unknown data. Five fold cross validation with $\mathrm{m}=5$ has been followed for classifier design in this work.

To evaluate the performance of a classifier following parameters are derived based on the results of classifier for validation data set.

$$
\begin{gathered}
\text { Sensitivity }=\frac{T_{P}}{T_{P}+F_{N}} \\
\text { Specificity }=\frac{T_{N}}{T_{N}+F_{P}} \\
\text { Accuracy }=\frac{T_{P}+T_{N}}{T_{P}+T_{N}+F_{P}+F_{N}}
\end{gathered}
$$

where, $T_{P}$ is number of True Positive, $T_{N}$ is number of True Negative, $F_{P}$ is number of False Positive and $F_{N}$ is number of False Negative. A confusion matrix holds these true and false results of a classifier as shown in Table II.

TABLE II. CONFUSION MATRIX FOR BINARY CLASSIFIER

\begin{tabular}{|c|c|c|}
\hline \multirow{2}{*}{$\begin{array}{c}\text { True Class a pulse } \\
\text { signal belongs to }\end{array}$} & \multicolumn{2}{|c|}{ Classified by Classifier as } \\
\cline { 2 - 3 } & Positive (P) & Negative (N) \\
\hline Positive (P) & $\mathrm{T}_{\mathrm{P}}$ & $\mathrm{F}_{\mathrm{N}}$ \\
\hline Negative (N) & $\mathrm{F}_{\mathrm{P}}$ & $\mathrm{T}_{\mathrm{N}}$ \\
\hline
\end{tabular}

Sensitivity, Specificity and Accuracy of the classifier are derived from the confusion matrix. Sensitivity is the fraction of subjects with the disease that the test correctly classifies as positive. On the other hand specificity is the fraction of subjects without disease that the test correctly classifies as negative. Accuracy gives the total subjects correctly classified out of all the subjects under consideration.

\section{RESULTS AND DISCUSSION}

A pulse classifier has been designed with Linear as well as Quadratic discrimination function. The features derived from power spectral density for 18 normal and 8 abnormal pulse 
patterns are used to prepare an overall data matrix of size Nxn; where ' $\mathrm{N}$ ' is 26 and ' $\mathrm{n}$ ' is 40 giving initial 0 to $10 \mathrm{~Hz}$ frequency components. To emphasis the real need of ranking the frequency domain features, Linear and Quadratic classifiers are trained with raw frequency domain features derived from power spectral density of the pulse series. Since the resolution of frequency components is $0.244 \mathrm{~Hz}$, the frequency range of $0 \mathrm{~Hz}$ to $10 \mathrm{~Hz}$ will require 40 initial frequency components from power spectral density. Grouping of these frequency components in sequence from $1^{\text {st }}$ to $40^{\text {th }}$ components is formed to prepare feature vector. The feature vector is used for training both Linear and Quadratic classifiers. The accuracy of both the classifiers for sequential grouping of the frequency components as feature vector is shown in Fig. 6. The accuracy of Linear and Quadratic classifier in this manner has found to be $76.20 \%$ and $74.40 \%$. The number of frequency components required to achieve these results are 12 and 5 respectively for Linear and Quadratic classifiers.

In an attempt of searching frequency components of prime importance for classification task, they are ranked based on their classification power. The initial ten ranked frequency components in the range of $0 \mathrm{~Hz}$ to $10 \mathrm{~Hz}$ are shown in Table III. The groups of such 25 ranked frequency components are used to train the classifier. The overall data base, after reducing their dimension for ranked frequency components, is divided in to training and test database. Linear and Quadratic classifiers are trained using 5 fold cross validation technique. The 5 fold cross validation results are averaged and similar procedure is repeated for 25 times. The average classification accuracy is derived for both the classifiers. Fig. 7 shows percentage accuracy for the test data set for both the classifiers for different group of initial 25 ranked frequency components.

Initially as frequency components are increasing in the feature vector, the test accuracy improves. After some stage, the test accuracy of both the classifiers falls with the increasing frequency components. When the weights are getting trained with large amount of features, classification accuracy for test data set reduces due to overfitting. The solution derived by the classifier as a part of weight learning procedure, results in a very specific solution, when classifier is trained with large amount of features. The weights thus obtained lead to over fitting and the test accuracy reduces due to lack of generalization capability of the classifier. Hence classifier gives better results for unknown data set for optimum ranked features where highest accuracy is achieved.

The highest classification accuracy achieved for Linear and Quadratic classifier is $89.0 \%$ and $89.2 \%$ for 5 and 4 initial ranked features. The parameters that can help in examining the performance of a classifier are shown in Table IV.

TABLE III. OPTIMUM FREQUENCY COMPONENTS AS RANKED FEATURES

\begin{tabular}{|c|c|c|c|c|c|c|c|c|c|c|}
\hline \multirow{2}{*}{$\begin{array}{c}\text { Ranked } \\
\text { Parameter }\end{array}$} & \multicolumn{10}{|c|}{ Frequency Ranks } \\
\hline & 1 & 2 & 3 & 4 & 5 & 6 & 7 & 8 & 9 & 10 \\
\hline Frequency & 0.97 & 1.22 & 8.05 & 6.83 & 8.30 & 2.68 & 2.92 & 9.76 & 4.15 & 9.27 \\
\hline
\end{tabular}

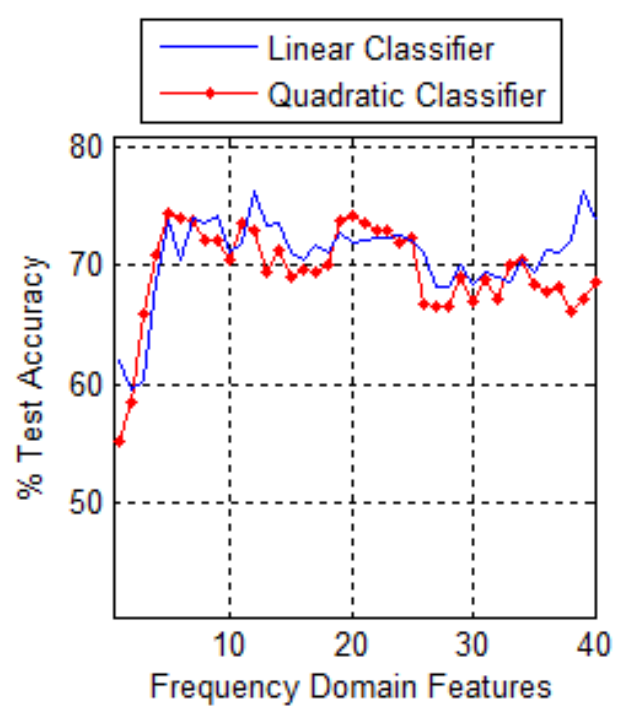

Figure 6 Test Accuracy in absence of ranked features

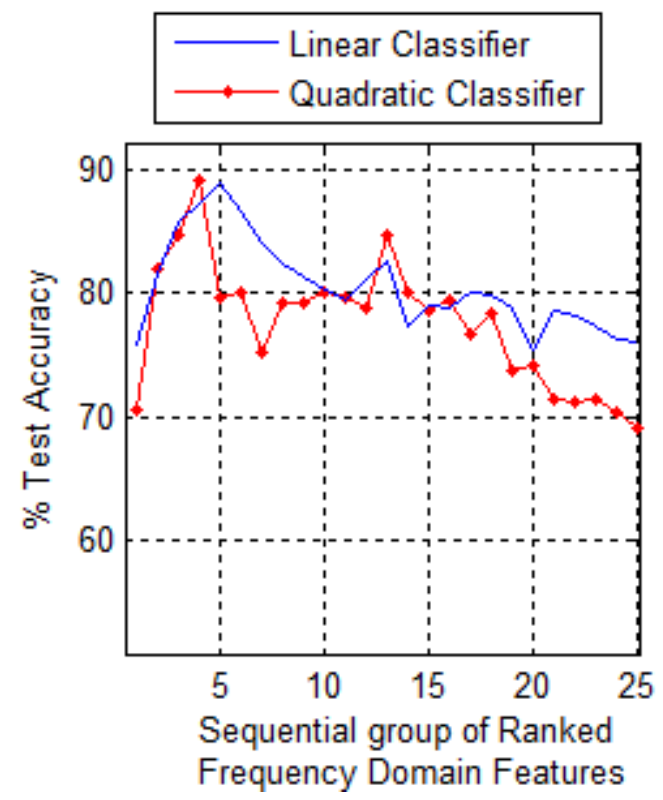

Figure 7 Test Accuracy for group of ranked features

TABLE IV. PERFORMANCE MEASURES FOR CLASSIFIERS

\begin{tabular}{|c|c|c|c|c|}
\hline \multirow{2}{*}{$\begin{array}{c}\text { Performance } \\
\text { Measure }\end{array}$} & \multicolumn{2}{|c|}{$\begin{array}{l}\text { Without Ranked } \\
\text { Features }\end{array}$} & \multicolumn{2}{|c|}{ With Ranked Features } \\
\hline & $\begin{array}{c}\text { Linear } \\
\text { Classifier }\end{array}$ & $\begin{array}{l}\text { Quadratic } \\
\text { Classifier }\end{array}$ & $\begin{array}{c}\text { Linear } \\
\text { Classifier }\end{array}$ & $\begin{array}{l}\text { Quadratic } \\
\text { Classifier }\end{array}$ \\
\hline$\%$ Sensitivity & 86.80 & 82.44 & 96.77 & 97.06 \\
\hline$\%$ Specificity & 54.32 & 55.75 & 71.48 & 73.72 \\
\hline$\%$ Accuracy & 76.2 & 74.4 & 89.0 & 89.2 \\
\hline $\begin{array}{c}\text { No. of Optimum } \\
\text { Ranked } \\
\text { Features }\end{array}$ & 12 & 5 & 5 & 4 \\
\hline
\end{tabular}

\section{CONCLUSION}

The frequency domain features derived from the power spectrum of the pulse series show significant information in the band of $0 \mathrm{~Hz}$ to $10 \mathrm{~Hz}$. There exists redundancy for the frequency components due to the fact that only very few frequency components are enough for classification task. In the absence of information about such frequency components, the classifier getting trained with raw frequency components results not only in poor accuracy but also require higher amount of frequency components. This ultimately raises the complexity of the classifier. 
With the help of a task of ranking the frequency components according to their classification power helps not only in achieving the dimensionality reduction but also improves the accuracy. The reduction in dimensionality of the feature vector ultimately reduces the complexity of the classifier. Looking at the results mentioned in Table IV, Quadratic classifier gives $89.2 \%$ classification accuracy with $97.06 \%$ sensitivity and $73.73 \%$ specificity for only 4 initial ranked frequency components mentioned in Table III.

The proximal pulse point for left hand showing dicrotic notch suppression at deep pressure in case of subjects suffering from prostate enlargement proves the importance of this pulse point for UTI related problems. The optimum features selected from power spectrum of the pulse help in designing a simple Quadratic classifier with only 4 frequency domain features giving $89.2 \%$ accuracy to segregate an abnormal pulse with suppressed dicrotic notch from healthy pulses.

\section{ACKNOWLEDGMENT}

Bhaskar Thakker and Anoop Lal Vyas are thankful to New Delhi Municipal Council (NDMC), India for providing facilities for acquiring radial pulse signals. Partial support from a collaborative project under the UK India Education Research Initiatives (UKIERI) is also thankfully acknowledged.

\section{REFERENCES}

[1] B. Flaws "The Secrets of Chinese Pulse Diagnosis", 1995 Blue Poppy Press, Boulder, CO.

[2] Wang, Shu-ho., "The Pulse Classic", 1997 Blue Poppy Press, Boulder, $\mathrm{CO}$.

[3] L S Xu, K Q Wang, L Wang, "Pulse waveforms Classification Based on Wavelet Network" Proceedings of the $27^{\text {th }}$ Annual Conference IEEE Engineering in Medicine and Biology, 2005; pp. 4596-4599.

[4] L U Wang, Kuan-Quan Wang, Li-Sheng Xu "Recognizing Wrist Pulse Waveforms with Improved Dynamic Time Warping Algorithm" Proceedings of the Third International Conference on Machine Learning and Cybernetics, Shanghai, 2004; pp. 3644 -3699.

[5] L S Xu, Max Q H Meng, K Q Wang "Pulse Image Recognition Using Fuzzy Neural Network" Proceedings of the $29^{\text {th }}$ Annual International
Conference of the IEEE Engineering in Medicine and Biology, France, 2007; pp.3148 - 3151

[6] Chun Lee, Ling Wei, "Spectrum Analysis of Human Pulse", IEEE Transactions on Biomedical Engineering, 1983, pp. 348 - 351

[7] Ling Wei, Peter Chow, "Frequency Distribution of Human Pulse Spectra", IEEE Transactions on Biomedical Engineering, 1985; pp.245-246.

[8] Yu Ji-chun, Pan Zu-shan, Ni Zhen-qun, "Harmonic Analysis Method of Human Pulse Signal" 10th International conference of IEEE Engineering in Medicine and Biology, 1988; pp.263-264.

[9] Wan-An Lu "Pulse Spectrum Analysis in 205 Patients with Abnormal Liver Function Test", Taipei City Med J, 2006; pp. 47-53.

[10] Bhaskar Thakker, Anoop Lal Vyas and D M Tripathi "Radial Pulse Analysis at Deep Pressure in Abnormal Health Conditions" Third International Conference on BioMedical Engineering and Informatics, October, 2010; pp. 1007-1010.

[11] Dawber TR, Thomas HE and Jr, McNamara PM. "Characteristics of the dicrotic notch of the arterial pulse wave in coronary heart disease", Angiology, April, 1973; pp. 244-255.

[12] R. Duda, P. Hart, and D. Stork, Pattern Classification, second ed. New York: John Wiley and Sons, 2000.

Bhaskar Thakker obtained Bachelor of Engineering in Electronics in 1997 from Birla Vishwakarma Mahavidyala, Vallabh Vidhya Nagar, Gujarat, India. He obtained Masters of Engineering in Microprocessor Systems and Applications in 2000 from M.S. University, Baroda, Gujarat, India.

Since 1999 he has been lecturer and at present Associated Professor in Electronics and Communication Engineering Department at G H Patel College of Engineering and Technology (GCET), Vallabh Vidhya Nagar, Gujarat, India. Presently he is a research scholar in Instrument Design Development Centre at Indian Institute of Technology (IIT) Delhi, India.

Anoop Lal Vyas obtained Bachelor of Technology in Electrical Engineering in 1972 and Ph.D. in 1989 both from Indian Institute of Technology (IIT), Delhi.

Since 1972 he has been working at IIT Delhi in the areas of Sonar Signal Processing, Underwater Electronics and Electronic Systems and has co-coordinated a number of sponsored projects in these areas. He presently holds a position of Professor and his interests include Electronic Instrumentation, Smart Sensors, Sensor Networks and Telemedicine. He is also one of the coordinator of Indo-UK collaborative project on "Mobile Communications for improving monitoring of Heart Disease and Diabetes" under the UKIERI program of British Council. 